\title{
Is portal vein arterialization a possible therapeutical approach for acute hepatic failure?
}

\begin{abstract}
Mortality for Acute Hepatic Failure (AHF) is very high if a Liver Transplant (LT) is not available in the short time that passes from the deteriorating liver function and death. ${ }^{1}$ Portal Vein Arterialization (PVA) is a promising technique to fast the regeneration process and to reverse liver necrosis ${ }^{2-4}$ but his role in AHF need to be defined. We report a case of a 24 years old man with AHF due to drug assumption in whom PVA was effective to restore liver function also if the patient died 6days after operation for sepsis.
\end{abstract}

Keywords: liver function, liver necrosis, jaundice, doppler ultrasound, arterialization
Volume 2 Issue 4 - 2016

\author{
Recordare A,' Gogilashvili L, ${ }^{2}$ Otar Filishvili \\ $\mathrm{SB},{ }^{3}$ Sophio Beridze $\mathrm{S}^{4}$ \\ 'Division of Surgery, Dell'Angelo Hospital, Italy \\ 2Personalized Medical Institute, Georgia \\ ${ }^{3}$ Israeli Georgian medical research clinic Helsicore, Georgia \\ 4Department of Therapy, Batumi Referral Hospital, Georgia
}

Correspondence: Alfonso Recordare, IV Division of Surgery, Treviso Regional Hospital, Italy, Email alf.recordare@gmail.com

Received: August 2I, 2016 | Published: November 01, 2016
Abbreviations: AHF, acute hepatic failure; LT, liver transplant; PVA, portal vein arterialization; PA, prothrombin activity

\section{Introduction}

First introduced in 1992 like a method to replace a damaged hepatic artery during major hepatobiliary surgery, Portal Vein arterialization (PVA) is becoming now a method to rescue the grafts in the field of liver transplantation, when an arterial thrombosis occur, and there are no alternative ways to restore the arterial flow. Moreover there are a lot of experimental studies showing that PVA has a beneficial effect in many pathologic situations, like it is, for example, in the toxic liver or when there is a small remnant after resection. We report a case in which PVA was used in a patient with acute hepatic failure from toxic liver.

\section{Case report}

A 24years old man was admitted in Hospital after 4days of mild fever and malaise for the onset of jaundice. Lab tests revealed AST 2980U/1, ALT 1687U/I, total bilirubin 138mmol/L INR 3.8 . The clinical conditions progressively worsened and after 2 days the patient developed encephalopathy and coma with a Prothrombin Activity (PA) of $15 \%$, INR 8.7 , total bilirubin $235 \mathrm{mmol} / \mathrm{L}$. A viral etiology was excluded and a toxic etiology was supposed based on the medical history of recreational use of oral drugs. Abdominal ultrasound revealed a normal volume of the liver with patent vessels. After 2days of coma the patient was proposed for evaluation to our Centre. In front of the impending death, considered the unavailability of a graft for transplant, we tried to rescue the liver with PVA. MR excluded significant brain edema. A liver biopsy on segment $\mathrm{V}$ was taken (Figure 1) and an anastomosis between the inferior mesenteric vein and artery was completed (Figure 2). A good thrill was present at the end of operation. In the following days Doppler ultrasound showed the typical turbulent flow in the PV and a mild ascites. The lab test of the patient significantly improved in the next days after the operation (Table 1) while the GCS passed from 3 to 5. Anyway, after 4days, the general condition of the patient worsened again in spite of the improving liver function and the patient eventually died 6days after surgery in a clinical picture of septic shock. The results of blood cultures (available after death) revealed E. coli, Staphylococcus aureus and Candida resistant to all administered antibiotics. Consent for autopsy was denied from relatives.

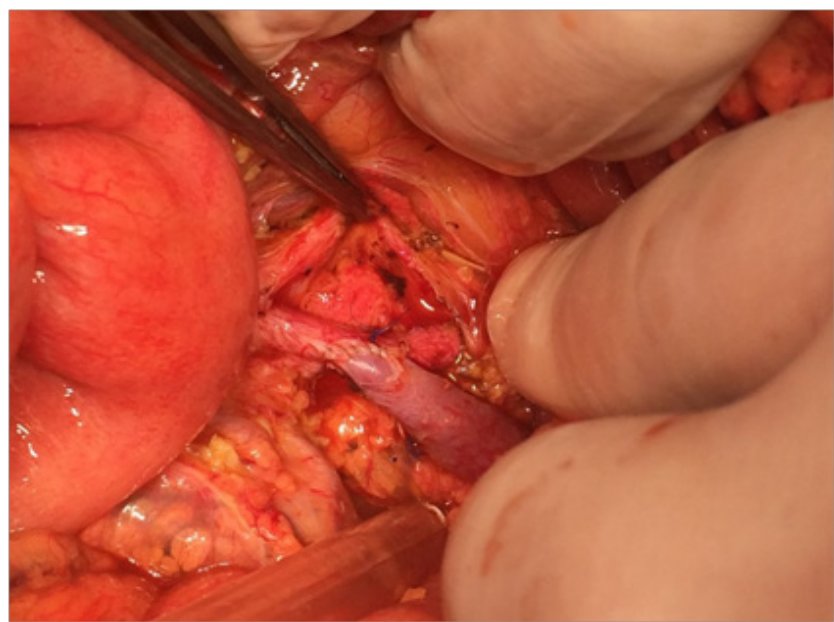

Figure I liver biopsy on segment $\mathrm{V}$.

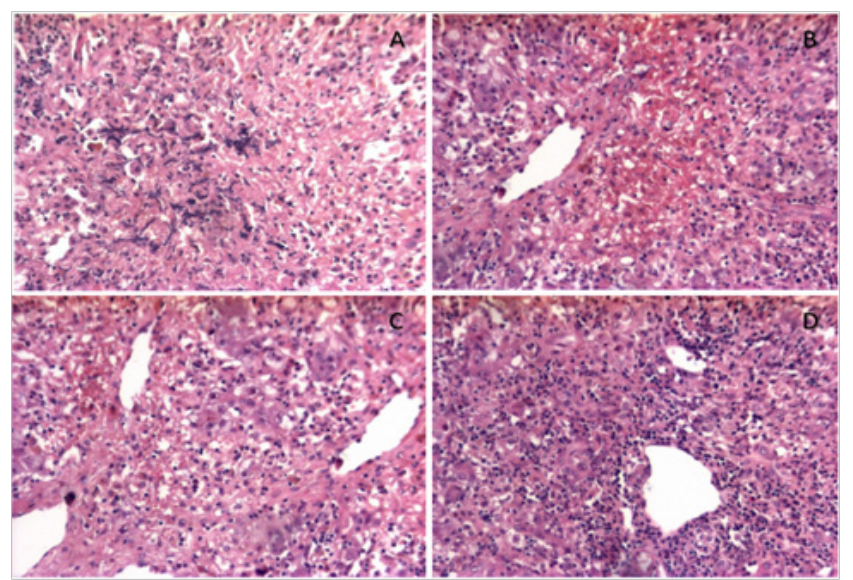

Figure 2 Anastomosis between the inferior mesenteric vein and artery and typical turbulent flow in the PV and a mild ascites. 


\section{Discussion}

First introduced in 1992 like a method to replace a damaged hepatic artery during major hepatobiliary surgery, ${ }^{3} \mathrm{PVA}$ is becoming now a method to rescue the grafts in the field of liver transplantation, when an arterial thrombosis occur and there are no alternative ways to restore the arterial flow. ${ }^{2}$ During these experiences it appeared evident that the Arterialization is not just useful to replace the hepatic artery flow, but also to reverse the necrosis that very often is linked to the thrombosis. The first report focusing on this beneficial effect was published in New Engl Med Journal on 2001,4 in a case with $80 \%$ liver necrosis on biopsy. This technique is gaining popularity in the transplant centres because it offers an easy solution in some dramatic situations. Not surprisingly, every group that introduced PVA in his armamentarium started to use the technique more and more widening the indications. The broad spectrum of indications to PVA is reported in a recent paper from the group of Paul Brousse. ${ }^{2}$

AHF is a life-threatening condition due to different causes and LT is the only effective therapy in patients with poor prognostic factors. ${ }^{1}$ In the toxic liver the target of therapy is to reverse the necrosis. Many experimental studies showed that also if the Arterialization has not a significant effect to modify the regeneration process in the normal liver, it has a beneficial effect in the damaged liver like it is for example when a toxic damage is induced from $\mathrm{CCl}_{4}{ }^{5}$
The demonstration that PVA is a useful technique:

i. In the field of transplantation when a graft is failing because of the impossibility to restore the flow in the hepatic artery.

ii. After a major liver resection that require an arterial resection ${ }^{6}$ and

iii. To promote liver regeneration in patient with a small remnant after major liver resection.

iv. To reverse massive necrosis ${ }^{2}$ or severe toxic injury of the liver would lead to think about a possible role in AHF. Anyway because of the polarization of surgeons and hepatologists toward the transplant, this possible therapeutical approach to AHF remains still in the shadow.

In our case, although the massive liver necrosis, PVA resulted in a significant improvement in liver function tests (Table 1). In spite of this improvement, patient developed sepsis, from whom he died 6days after surgery. Sepsis is a common event in FHF patients, especially after prolonged intubation, and remains the first cause of death in transplanted patients with functioning graft. ${ }^{1}$ Stated that this operation was performed very late, when a possible spontaneous recovery of this patient from AHF was extremely unlikely, it appears more evident that PVA worked to recover the liver function.

Table I Blood tests parameters and neurological status before and after portal vein Arterialization

\begin{tabular}{|c|c|c|c|c|c|c|c|}
\hline \multirow{2}{*}{ Parameters(Normal range) } & \multirow{2}{*}{ Preop Day - I } & \multicolumn{6}{|c|}{ Post PVA } \\
\hline & & Day +I & Day +2 & Day +3 & Day +4 & Day +5 & Day +6 \\
\hline PT \%(70-100) & 20 & 15 & 23 & 35 & 37 & 45 & 21 \\
\hline INR $(I-I, 15)$ & 6,23 & 3.9 & 2.5 & 1.9 & 1.8 & 1.5 & 2.6 \\
\hline Total Bilirubin $(<26 \mu \mathrm{mol} / \mathrm{L})$ & 235 & 278 & 256 & 280 & 219 & 192 & 220 \\
\hline AST (0-35 U/L) & 2840 & 1 & 235 & 467 & 414 & 215 & 135 \\
\hline ALT (3-36 U/L) & 1480 & 266 & 272 & 202 & 204 & 139 & 108 \\
\hline Creatinine $(70-120 \mu \mathrm{mol} / \mathrm{L})$ & 120 & 110 & 73 & 134 & 127 & 144 & 206 \\
\hline GCS & 3 & 3 & 3 & 5 & 5 & 3 & 3 \\
\hline Ammonia (I l-35 $\mu \mathrm{mol} / \mathrm{L})$ & 367 & 289 & & & 165 & & \\
\hline $\mathrm{pH}$ & 7.2 & 7.4 & 7.4 & 7.4 & 7.5 & 7.4 & 6.9 \\
\hline
\end{tabular}

PVA, portal vein arterialization; PT\%, prothrombin time (percentage of the standard value); Cre, creatinin; GCS, glasgow coma score.

Regarding the technical aspects, previous reports ${ }^{2}$ show that it's preferable to use medium size arterial inflow vessels, to ensure good oxygenation and to increase the chance of patency. In our patient we took in account the marked coagulopathy, that make advisable to use a site that do not require extensive dissection to perform the shunt, through a short midline incision that doesn't require an interposition graft and can be performed in a short time. This approach has also has the advantage to leave intact the hepatic hylum and major vessels in case of future operations and easily reversed by embolization or in any case after eventual recovery ${ }^{2}$ or the onset of complications due to portal hypertension.

\section{Conclusion}

This report seems to confirm that PVA can be effective to reverse massive liver necrosis in toxic acute liver failure. We think that this approach needs to be taken in consideration in patient with so poor prognosis especially in countries where a liver transplant is not easily available. Further experiences are needed to understand the role of PVA in the treatment of AHF, the timing of the procedure and the integration with other available techniques.

\section{Acknowledgements}

None.

\section{Conflict of interest}

Author declares that there is no conflict of interest.

\section{References}

1. Farmer DG, Anselmo DM, Ghobrial RM, et al. Liver Transplantation for Fulminant Hepatic Failure: Experience With More Than 200 Patients Over a 17-Year Period. Ann Surg. 2003;237(5):666-675. 
2. Morimoto T, Terasaki M, Higashiyama $\mathrm{H}$, et al. Clinical application of arterialization of portal vein in living related donor partial liver transplantation. Transpl Int. 1992;5(3):151-154.

3. Bhangui P, Salloum C, Lim C, et al. Portal vein arterialization: a salvage procedure for a totally de-arterialized liver. The Paul Brousse Hospital experience. HPB (Oxford). 2014;16(8):723-738.

4. Cavallari A, Nardo B, Caraceni P. Arterialization of the portal vein in a patient with de-arterialized liver graft and massive necrosis. $N$ Engl $J$ Med. 2001;345(18):1352-1353.
5. Nardo B, Puviani L, Caraceni $\mathrm{P}$, et al. Successful treatment of $\mathrm{CCL}_{4}$ induced acute liver failure with portal vein arterialization in the rat. Transplant Proc. 2006;38(4):1187-1189.

6. Shimizu Y, Miyazaki M, Shimizu H, et al. Beneficial effects of arterialization of the portal vein on extended hepatectomy. Br J Surg. 2000;87(6):784-789.

7. Nardo B, Montalti R, Puviani L, et al. Portal vein arterialization in a patient with acute liver failure. Transplantation. 2005;79(7):851-852. 